\title{
Review
}

\section{Texture and Rheology in Food and Health}

\author{
Katsuyoshi NiSHINARI* \\ Department of Food and Human Health Sciences, Graduate School of Human Life Science, Osaka City University, 3-3-138 Sugimoto, \\ Sumiyoshi-ku, Osaka 558-8585, Japan
}

Received October 27, 2008; Accepted December 6, 2008

The importance of texture control in food and health in Japan was discussed on the occasion of the celebration of the $100^{\text {th }}$ anniversary of Japanese immigration to Brazil. Dietary patterns in both countries, recent research activities, and governmental recommendation of food intake are also described, including some historical changes. Recent advances in study on texture terms, functional foods, food gels, and gelling process in the food science and technology in Japan are also reviewed.

Keywords: hydrocolloids, gel, texture, rheology, language, mastication, deglutition

\section{Introduction}

Along with flavor, aroma, appearance, and color, texture is one of the most important determining factors for the palatability of foods, and therefore many studies have been conducted on the texture of foods. The importance of texture and the wide-ranging research are the reasons why the $1^{\text {st }}$ Food Summit in 1999, held in The Netherlands, chose the title "Texture - Perception and its measurements." As defined by Szczesniak (2002), texture is a sensory property, and can be detected only by humans. Sensory organs of humans are not as sensitive as modern electronic sensors, which, for example, can detect even a very small length on the nano scale. Properties that cannot be detected by humans are not textures, although nanoscale structure may constitute some perceivable textures.

Recent changes in our modern lifestyles have led to increasing demand for convenience foods, and our growing awareness of the relationship between food and health has increased the demand for higher-fiber, lower-fat products. In 2005, the Japanese Ministry of Agriculture, Forestry and Fisheries, in collaboration with the Ministry of Health, Labour and Welfare, worked out the Balance Guide for Diet (http://www.maff.go.jp/food_guide/balance.html), which recommends well-balanced intake of cereals, vegetables, mushrooms, tubers, seaweeds, meat, fish, eggs, soybeans, milk and dairy products, and fruits.

*To whom correspondence should be addressed.

E-mail: nisinari@life.osaka-cu.ac.jp
Meanwhile, to satisfy demands of the people, texture is important to make foods more palatable, and texture control has also become even more important for creating safe, pleasant foods for the elderly who have difficulty in mastication and deglutition (Nishinari, 2004). Since pneumonia induced by silent aspiration is the leading cause of the death among the elderly, many doctors and nursing care workers pay close attention to prevent silent aspiration and to take care of persons with dysphagia. As has been found empirically, thickening of thin drinks such as tea or fruit juice is effective in preventing silent aspiration (Logemann, 1983). Texture studies of foods, therefore, are becoming more important, to make foods more palatable and safer, to allow transfer into digestive organs without causing silent aspiration and gastroesophageal reflux disorder.

\section{Texture terms}

Japanese people are fond of a variety of textures, and therefore many terms for texture terms have come into use. About 40 years ago, Yoshikawa and his colleagues collected approximately 450 Japanese texture terms and classified them into four categories (Yoshikawa et al., 1970). The characteristics of Japanese texture terms are that there are many examples of onomatopoeia; i.e., words that mimic a sound, as well as words that represent the mode or appearance when foods come into contact with organs in the oral cavity, or when solid foods are deformed or when liquid foods flow. Recently, Hayakawa et al. (2005) collected Japanese texture terms, and compared them with those used 40 years ago. 
They found that some core terms are still used, some terms used previously are not used very often, and some new terms have been introduced with the appearance of new processed foods such as new dessert jellies and fizzy drinks.

Recently, many foods have been imported to Japan from not only China but also various other countries, as Japan's self-sufficiency rate is very low $(<40 \%)$. In this age of globalization, understanding words from different languages representing textures of foods is becoming more and more important. Therefore, comparing texture terms in different languages is useful for both academic researchers and workers in trade and industry. English, French, Japanese, and Chinese texture terms were compared, and in all four of these languages, the number of texture terms concerning solid foods was found to be much larger than that for liquid foods (Nishinari et al., 2008).

Extending this study to more languages, including Portuguese, is desired, as diversity is very important in foods. Needless to cite the episode of manna from heaven in the Old Testament, human beings do not feel happy if they are forced to eat only one kind of food, even if it is delicious and nutritious.

\section{Characteristics of Japanese diet}

As reviewed by the present author about 20 years ago (Nishinari, 1987), the Japanese dietary pattern has been believed to be closely related to the longest life expectancy in the world. McGovern's committee concluded that reducing fat intake is necessary to prevent chronic and life-style related diseases such as cancer and diabetes. Japanese people are fish-eating people. The fish catch was the largest before 1990 when fish production in China exceeded that of Japan, and the importation of fish has also been important for Japan.

Rice has been a staple food in Japan. On the occasion of a poor crop in 1993, Japanese people who did not know how to cook indica-type rice imported from Thailand threw the imported rice away, causing a scandal. Japanese people prefer Japonica-type rice boiled in Japanese style, and do not accept rice cooked in other ways. Rice cooked in Japanese style should have appropriate stickiness and elasticity. This preference explains the high popularity of japonica rice, koshi-hikari, sasa-nishiki, and akita-komachi (Matsuo et al., 2002, Okadome et al., 1998).

Soybeans have been one of the most important food ingredients in Japan, being the main ingredient of tofu, natto, miso, yuba, aburage, atsuage, etc., which constitute important protein sources in the Japanese diet. Westernization of the Japanese diet has shifted the traditional Japanese diet to higher intake of fat and oil, which has been identified as a cause of high hyperlipemia, diabetes, and other lifestyle- related diseases. Fuji Foundation for Protein Research has been promoting research on soybean protein in Japan. (http:// www.fujioil.co.jp/daizu/index.html)

Mushrooms have been favorite foods in Japan, and polysaccharides extracted from mushrooms have attracted much attention because of their beneficial physiological functions. Antidiabetic effect of an acidic polysaccharide from Tremella aurantia has recently been reported (Kiho et al., 2001). $\beta-1,3$ glucans, important ingredients of various mushrooms, have been studied mainly for their anti-tumor activity. Curdlan is a $\beta-1,3$ glucan without side chain, discovered by Harada of Osaka University (Harada et al., 1966), and has been applied to production of various foods such as frozen tofu with smooth texture and for gel-like foods (Nishinari, 1987; Nishinari and Zhang, 2000). Some new developments in physicochemical aspects of $\beta-1,3$ glucans, including those from cereals, are described in the second edition of Chemistry, Biochemistry, and Biology of $\beta-1,3$ glucans (Gidley and Nishinari, 2009).

Seaweeds such as wakame, konbu, and hijiki have been important foods in Japan. Agar and carrageenan are used for sweet desert jellies and in other recipes. Gelling behaviors of polysaccharides from seaweeds have recently been reviewed (Nishinari, 2005; Shiba and Nishinari, 2007).

\section{Gel-like foods}

Surimi, fish paste, and its various forms, kamaboko, hanpen, and chikuwa, are traditional Japanese foods. According to statistics of producers, their consumption has gradually decreased, and the tough, rubber-like texture has tended to be avoided year by year. On the other hand, the crabstick, kaniashi kamaboko, was introduced in the market some 40 years ago; its consumption has steadily increased and it is now sold also in European countries and in America.

Tofu has been one of important foods in Japan, and it is now also appreciated in other Asian countries as well as in America. The aseptic packaging of $t o f u$ was introduced in the 1960's, which eliminated the need for usage of harmful synthetic preservatives such as AF2 and extended shelf life without relying on any preservatives. In North America, the dairy industry uses paper packaging for tofu. Yu-doufu, cooked in hot water, and hiya-yakko, cooled in ice water, have been among the favorite dishes in Japan. Tofu is also an important ingredient in sukiyaki, cooked with beef, leek, shirataki cooked in soy sauce (shoyu), and oden, cooked with konnyaku, boiled eggs, chikuwa, and daikon (mooli, Japanese radish). Okara, residue remaining after the production of soy milk, has been eaten in traditional dishes, but most okara is now transported for incineration, since the consumption of okara is not very high and it cannot be stored for a long time. 
This remains an important problem to be solved. Some research has been conducted toward utilizing okara, adding it to breads (Uwabe et al., 2001), and so on. Recently, tofu was prepared without removing okara and was evaluated quite well (Yoshimura et al., 2004, 2007) which is good news for protecting the environment and is also good for health, because okara has a high content of dietary fiber.

Imitation $i k r a$, salmon roe, was invented more than 20 years ago, and has been used even for sushi. Ikra was originally made from salad oil, carrageenan or xanthan, alginate, and some flavors (Nishinari, 1987). A similar spherical particle can be formed easily by dribbling, dropwise, an aqueous solution of gellan or alginate into a calcium chloride solution (Fig. 1). Doing so has proven to be a popular demonstration for high school students who visit our university on open campus day during summer vacation.

Konjac, konnyaku in Japanese, has been a traditional food ingredient in Asian countries, including Korea and China. The most common form of this gel has been a so-called itakonnyaku, a gel molded into a plate of $2 \mathrm{~cm}$ thickness, a length of $20 \mathrm{~cm}$, and a width of $10 \mathrm{~cm}$. It has a peculiar rubber-like texture, and has also been changed into another form such as ito-konnyaku or shirataki, noodle-type gel. Sometimes these konjac strings are twisted into a rope-like form of about $1 \mathrm{~cm}$ diameter, which appeared in the market some 10 years ago and sold well because, in comparison with a bulk block type konnyaku, it could easily catch flavor.

With the advent of the aged society, food is surely eaten to supply energy to the body, but it is also taken for enjoyment and health improvement. Polysaccharides play a role of dietary fiber and reduce blood cholesterol, and therefore attract attention in modern society. These factors have given rise to a considerable increase in the use of polysaccharides in food products. Our research group has studied the gelation of polysaccharides and proteins.

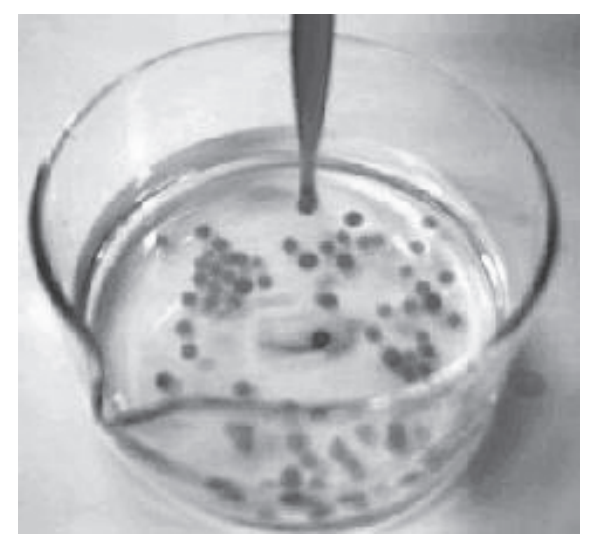

Fig. 1. Aqueous gellan solution containing red food dye drips down into $\mathrm{CaCl}_{2}$ solution, and forms a spherical droplet covered with a film. To make it palatable like ikra, some flavors are added.
Gelation and rheology of tofu, konnyaku, cheese, gellan, and starch

Gelation of soy protein has been studied by a dynamic viscoelastic method (Fig. 2, Nishinari et al., 1991). To understand the gelation mechanism, small-deformation rheology is useful because it can detect the rheological change during sol to gel transition without destroying the three-dimensional network being formed, whereas large-deformation rheology such as steady shear viscosity measurement destroys the structure. The gel formation of soy protein can be detected as increases in the storage and loss moduli, $G^{\prime}$ and $G^{\prime \prime}$, which represent elastic and viscous terms of the complex elastic modulus in shear mode, respectively. The same method was applied to understand the gelation mechanism of konjac glucomannan (Huang et al., 2002; Gao and Nishinari, 2004) and for rennet-induced gelation of casein micelles (Niki et al., 1994).

The gelation mechanism of konjac has been studied extensively by Maekaji et al. (Maekaji, 1978; Maekaji and Kawamura, 1984), and more recently by our group (Nishinari, 2000). The main ingredient of konjac is konjac glucomannan, KGM, consisting of $\beta$-1, 4-linked D-mannose and D-glucose with about one in 19 units being acetylated. KGM forms a thermally stable gel upon addition of alkaline coagulant and, in contrast to many other thermo-reversible cold setting gels such as agar gels or gelatin gels, heating promotes the gelation of KGM. Gelation occurs through the formation of a network structure of junction zones, which are considered to be stabilized by hydrogen bonding.

Gellan gum is a microbial polysaccharide, which is produced by Sphingomonas elodea, and consists of a tetrasaccharide unit, glucose, glucuronic acid, glucose, and rhamnose. It was first approved for food use in Japan in 1988.

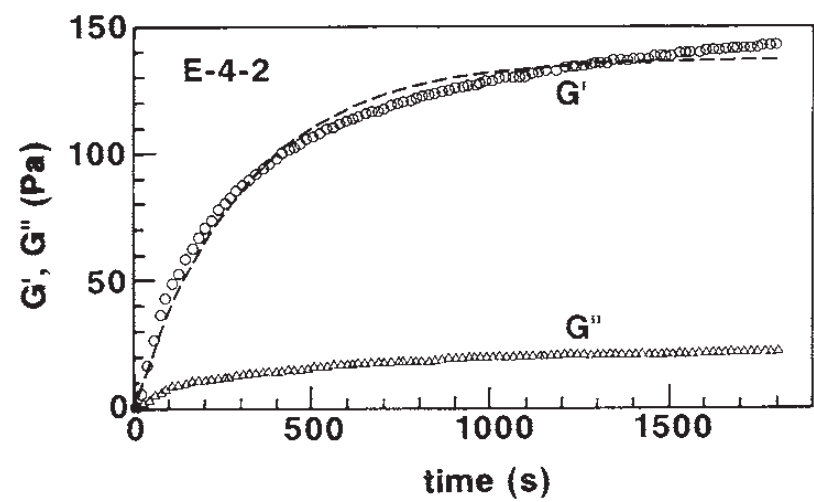

Fig. 2. Gelation process of soybean protein $(4.0 \mathrm{wt} \%)$ in the presence of $0.4 \%$ glucono-delta-lactone at $65^{\circ} \mathrm{C}$ (Nishinari et al., 1991). After addition of glucono-delta-lactone some time was required before the storage and loss moduli, $G^{\prime}$ and $G^{\prime \prime}$, began to increase. In this figure, time zero is taken as the time when $G^{\prime}$ and $G^{\prime \prime}$ began to deviate from the base line. 
Gellan gum has been expected to be not only a new gelling agent which forms a transparent gel resistant to acid and heat in the presence of divalent cations, but also a good model for the study of the thermoreversible gelling process, because it is dissolved well in water when converted into a sodium type or tetramethyl ammonium type, and it forms a transparent gel. A collaborative research group was organized in the Research Group of Polymer Gels affiliated with the Society of Polymer Science, Japan, in 1989. The group consists of various laboratories with different disciplines; rheology, nuclear magnetic resonance (NMR), electron spin resonance (ESR), differential scanning calorimetry (DSC), circular dichroism (CD), light scattering, osmotic pressure, small angle X-ray scattering, scanning tunneling microscopy, dielectric measurement, and so on. The results of the collaborative research were published as a special issue of Food Hydrocolloids in 1993, Carbohydrate Polymers in 1996, and Progress in Colloid and Polymer Science in 1999.

Figure 3 (a) shows temperature dependence of the storage and loss moduli, $G^{\prime}$ and $G^{\prime \prime}$, of 2 wt $\%$ TSX (tamarind seed xyloglucan) with $0.1 \mathrm{wt} \%$ EGCG (epigallocatechin gallate) on cooling and on subsequent heating, and Fig. 3 (b) shows cooling and heating DSC curves (Nitta et al., 2003). The
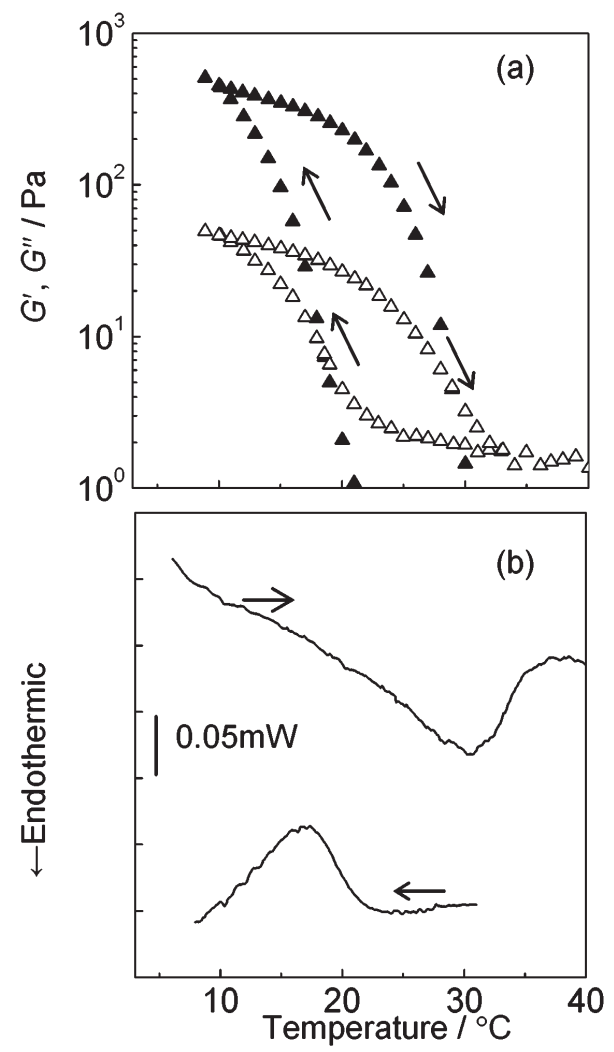

Fig. 3. (a) Temperature dependence of the storage and loss moduli, $G^{\prime}(\boldsymbol{\Delta})$ and $G^{\prime \prime}(\triangle)$ on cooling and on subsequent heating, and (b) cooling and subsequent heating DSC curves of $2 \mathrm{wt} \%$. TSX with $0.1 \mathrm{wt} \%$ EGCG. Scanning rate, $0.5{ }^{\circ} \mathrm{C} / \mathrm{min}$. Frequency, $1 \mathrm{rad} / \mathrm{s}$. Nitta et al. (2003).
TSX-EGCG gel was found to be thermoreversible. The storage modulus $G^{\prime}$ exceeded the loss modulus $G^{\prime \prime}$ around $20^{\circ} \mathrm{C}$ at $1 \mathrm{rad} / \mathrm{s}$, and thereafter $G^{\prime}$ and $G^{\prime \prime}$ increased with decreasing temperature. On subsequent heating, $G^{\prime}$ and $G^{\prime \prime}$ decreased steeply and $G^{\prime \prime}$ became larger than $G^{\prime}$ around $30^{\circ} \mathrm{C}$. These temperature-induced rheological changes were attributed to the gelation and the subsequent gel melting. DSC curves also supported the occurrence of the sol-gel transition. In cooling DSC curves, an exothermic peak appeared at almost the same temperature where $G^{\prime}$ and $G^{\prime \prime}$ increased steeply. An endothermic DSC peak appeared around the temperature where $G^{\prime}$ and $G^{\prime \prime}$ decreased steeply on heating. Thermal hysteresis was observed in both rheological and DSC results.

Yoshimura et al. (1999) found that the elastic modulus of cornstarch gels increased remarkably during storage for one or two weeks at $5^{\circ} \mathrm{C}$ but decreased to the initial level before storage by heating to $60^{\circ} \mathrm{C}$. Subsequent cooling did not recover the initial value of the elastic modulus, indicating that the network structure formed by amylopectin is easily disintegrated and is not recovered quickly (Fig. 4). The re-heating DSC curve for a starch gel stored for seven days showed a larger endothermic peak than for a gel stored for one day, and the peak temperature shifted to lower temperatures with increasing storage time, indicating that the recovered structure is more fragile thermally (Yoshimura et al., 1996). This finding is consistent with the pronounced temperature dependence of the elastic modulus shown in the right-hand figure of Fig. 4.

Effects of various polysaccharides: konjac, glucomannan, guar, and xyloglucan, on the gelatinization and retrogradation of starch have been investigated, and these polysaccharides are shown to be effective in retarding the retrogradation (Yoshimura et al., 1996; Funami et al., 2005; Pongsawatmanit et al., 2006).

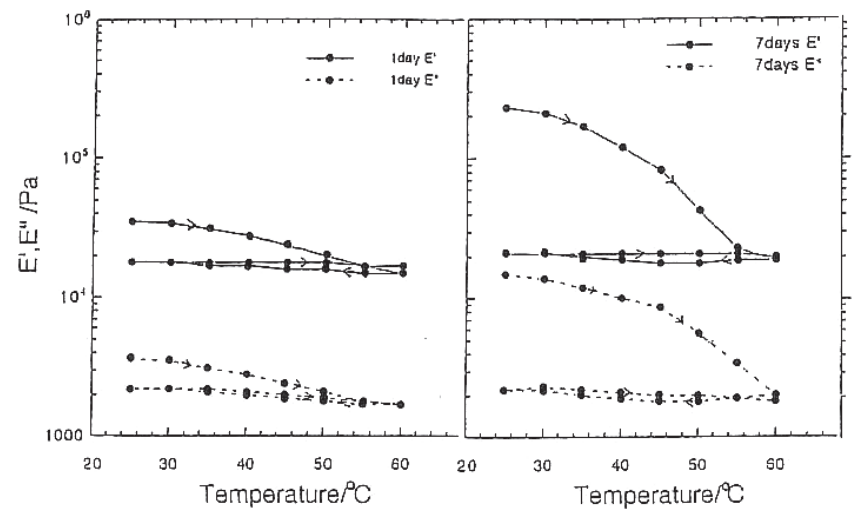

Fig. 4. Temperature dependence of storage and loss Young's moduli, $E^{\prime}$ and $E^{\prime \prime}$, of $20 \%$ corn starch gels stored at $5^{\circ} \mathrm{C}$ for one (left) and seven (right) days. Frequency: $3 \mathrm{~Hz}$, strain: 0.0033. (Yoshimura et al., 1999). 


\section{Mixture of different polysaccharides}

Since KGM interacts synergistically with carrageenan (Kohyama et al., 1993) or xanthan (Annable et al., 1994) even at lower $\mathrm{pH}$; for example, in the presence of fruit juices, konjac jellies have been produced and sold on a massive scale. They are packed in small plastic containers, and some unfortunate accidents have occurred when babies or elderly people swallowed konjac jelly and suffocated, although eating konjac jelly seems to be safe if care is taken with these persons.

Tamarind seed xyloglucan has been used as a thickening agent, because it is stable over a wide $\mathrm{pH}$ range and does not retrograde as starch does. When mixed with gellan (Nitta, et al., 2003) or xanthan (Kim et al., 2006), it forms a gel and is expected to be applied as a new texture modifier.

As shown in Figs. 5 (a) and 5 (b), $G^{\prime}$ and $G^{\prime \prime}$ of the mixture showed a steep increase at around $25^{\circ} \mathrm{C}$, whereas no such steep increase is found in Fig. 5(c). This steep change was attributed to the gel formation, and these results suggest that pyruvate groups in xanthan play a key role in the synergistic interaction.

\section{Large deformation and rupture of food gels}

We must understand the relation between large deformation and rupture behavior of food gels, but few quantitative studies have been published, due to mathematical and experimental difficulty. Recently, an analysis was conducted on strain hardening and the subsequent strain softening behavior of gellan gels containing sucrose in uniaxial compression measurements (Kawai et al., 2008). The rupture strain of a gellan gel containing sucrose is much larger than that of a gel without sucrose (Fig. 6). In the uniaxial compression of a gel at a moderate compression speed, $\lambda_{x}=\lambda_{y}=\lambda$ and $\lambda_{z}=\lambda^{-2}$.

The gel is assumed to consist of junction zones of helical aggregates and released-out chains from junction zones. When $v$ segments are released from the junction zone, the

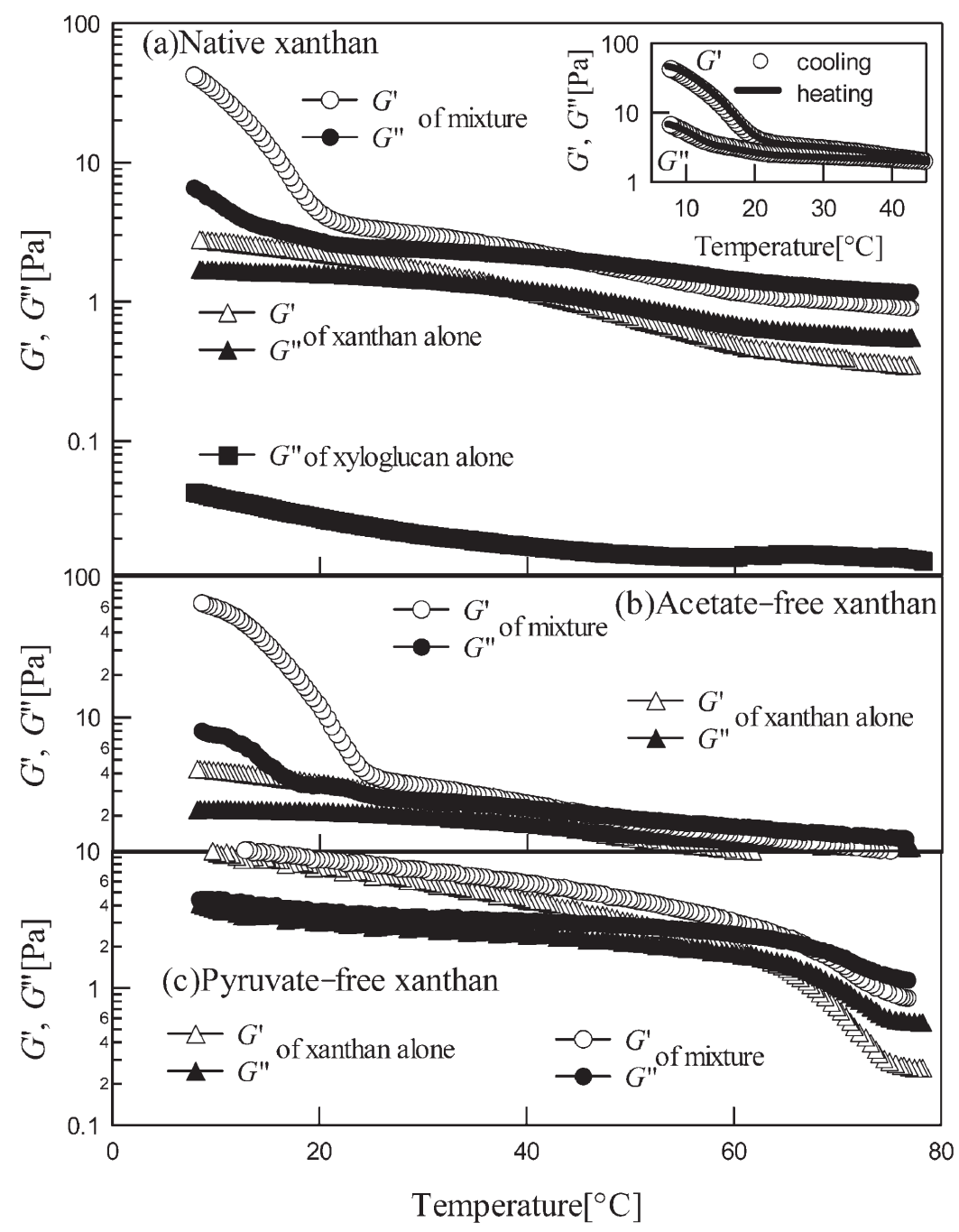

Fig. 5. Temperature dependence of the storage and loss moduli, $G^{\prime}$ and $G^{\prime \prime}$ of xanthan/xyloglucan mixture on cooling. Total polysaccharide concentration $1.0 \%$, xanthan $/$ xyloglucan $=$ 1/1, Cooling rate: $0.5^{\circ} \mathrm{C} / \mathrm{min}$, Angular frequency: $1.0 \mathrm{rad} / \mathrm{s}$. (Kim et al., 2006). 

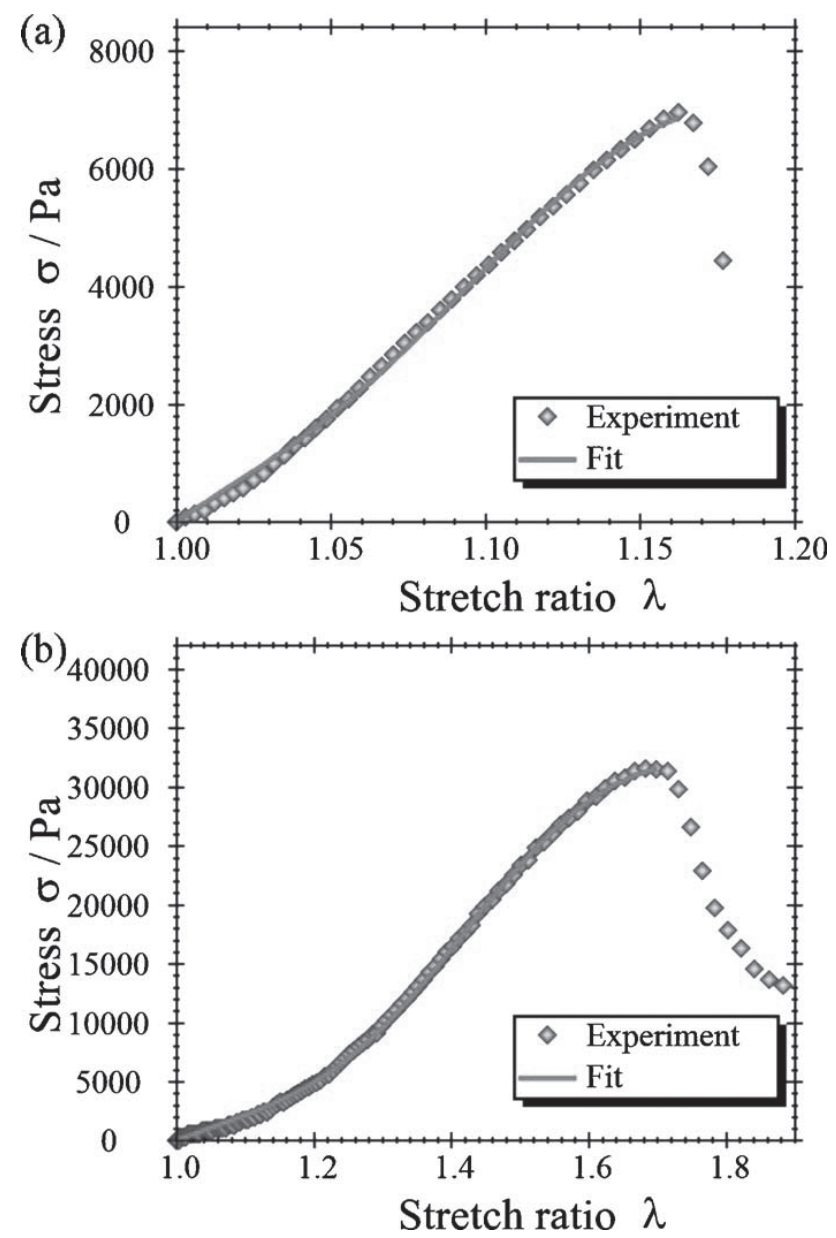

Fig. 6. (a) and (b) Stress and strain curves obtained by compression $(\diamond)$ and the calculated curves $(-)$ based on a modified reel-chain model (Kawai et al., 2008).

chain is liberated completely and becomes a dangling chain and therefore does not contribute to elasticity (Nishinari et $a l ., 1985)$. The average end-to-end distance is significantly shorter for sucrose-containing gels than for those without sucrose. As a result, sucrose-containing gels become highly flexible with yield strain reaching about 1.7, as contrasted with 1.15 for those without sucrose. The reduction of end-toend distance can be interpreted as an enhancement of crosslinking by addition of sucrose, if $v$ does not vary significantly. The enhanced cross-linking in the existence of sucrose is consistent with a previous finding (Miyoshi et al., 1998).

\section{Rheology, mastication, and deglutition}

The physiological texture measurements using sensors of force and deformation and position of the teeth and tongue, such as electromyography, electropalatography, and palatal pressure measurements, have been also introduced to simulate the masticating action better than uniaxial compression, and are summarized well in the textbook of Bourne (2002). Further endeavor to activate the interaction between food physics, psychology, and oral physiology continued, result- ing in publication of a Handbook of Creation of New Texture (In Japanese) (Nishinari et al., 2005).

The Japanese Society of Mastication was founded in 1990. After the Second World War, most processed foods became softer and softer, and the need for mastication became less and less. Many scientists pointed out the importance of mastication. Japanese research groups have tried to find scientific evidence to substantiate some beliefs and expectations for the important role of mastication in health, e.g., preventing Alzheimer's dementia. Momose et al. (1997) showed, by positron emission tomography, that mastication activated blood flow in the brain, and Onozuka et al. (2003) found evidence for the brain-stimulating action of mastication by comparing the memory of mice with and without molars. The First International Congress on Mastication and Health was organized by the Japanese Society for Mastication Science and Health Promotion (http://wwwsoc.nii.ac.jp/sosyaku/) in Yokohama in 2002. A special issue of Journal of Texture Studies was published in 2004 based on contributions of Bourne, Lucas, Bosman, Hiiemae, Mioche, and myself, who delivered lectures at a symposium of this congress (Nishinari, 2004)

To solve the problems in dysphagia, the rheological properties of foods have been controlled so that foods can be masticated and form a bolus suitable for swallowing. The bolus should have suitable rheological, colloidal, and surface properties for transfer to the esophagus and stomach. A "mouth process model" was proposed: structure of foods should be broken down and mixed with saliva to lubricate the foods sufficiently to form a bolus before swallowing (Hutchings and Lillford, 1988).

Videofluorography is being used for direct observation of the intake, mastication, and swallowing process of foods in human beings. Physicochemical properties of foods are sometimes influenced by contrast medium, such as barium sulfate, which is necessary to make foods visible by X-rays. For example, many kinds of gels are used to study deglutition, and the viscoelasticity of gels of polyelectrolytes such as gelatin, carrageenan, and gellan is changed drastically by the addition of divalent cations. To resolve this problem, a new method must be developed to detect the transport process of masticated foods, bolus. Recently, cine MRI was introduced to observe the swallowing process (Michiwaki et al., 2002).

\section{Food technology research in Japan}

Japanese Society of Food Science and Technology (JSFST, http://www.jsfst.or.jp/) was founded in 1953 and has been engaging in organizing annual meetings, lectures, and research seminars. Moreover, the Society presents academic 
awards with the objective of contributing to the advancement of food science and technology through cooperation among researchers, engineers, and other professionals in businesses, universities, and national and public research institutions. We currently have 3000 members, and publish two journals "Nippon Shokuhin Kagaku Kogaku Kaishi" (In Japanese) and "Food Science and Technology Research"(In English). Foods for Japanese astronauts (http://iss.jaxa.jp/spacefood/) are now being developed in Japan by collaboration between JSFST and JAXA (Japan Aerospace eXploration Agency) under the leadership of Prof. Tajima, since previous foods for astronauts were mainly made for American and Russian astronauts, and were not suitable for Japanese astronauts.

The life expectancies in Japanese communities in Hawaii and Brazil were surveyed around 2000 and were found to be 17 years shorter in Brazil than in Japan, which was attributed to the excessive intake of fat and salt, which led to arteriosclerosis and cardiac death (Yamori, 2007). Prof.Yamori also compared the incidence rate of diabetes in Japanese communities in Hawaii and Brazil, and found that during the Second World War the Japanese dietary pattern was followed more closely in Hawaii than in Brazil; especially, the intake of soybeans was very low in Brazil, which was confirmed by measuring isoflavone content in urine. Yamori came to Brazil in 1996 to examine the effect of isoflavone. Japanese immigrants in Brazil collaborated with him to carry out this test by taking isoflavone for three weeks. He concluded that isoflavone reduces blood pressure and cholesterol, which he explained by the estrogen-like function of isoflavone.

Physiological functions of various ingredients have been extensively studied over the past twenty years in Japan by Profs. Arai, Chiba, Osawa, and many active scientists. About 700 special foods are approved by the Ministry of Health, Labour and Welfare as being effective in reducing the risk of diet-related-diseases such as food allergy, obesity, and type two diabetes. These tokutei-hokenyou-shokuhin, or foods for specified health uses (FOSHU), have attracted much attention also in Europe and America. Nutrigenomics has become a powerful tool to examine the effect of nutrients on thousands of genes in the human genome. Using DNA microarray (genome chips), Prolla and his colleagues (Lee et al., 1999) showed that caloric restriction retards the aging process, and beneficial physiological functions of polyphenols contained in green tea, cocoa, herbs, and wine have been also demonstrated in Japan by these methods (Arai, 2007). Many nutritional scientists who study these problems also use cell culture experiments, animal testing, and epidemiological survey. JSFST, in collaboration with the Office of Food Technology Development, MAFF, supports the evaluation of food functionality (http://www.maff.go.jp/soshiki/syokuhin/kigyoush- inkou/sien.html).

A symposium on physical properties of foods has been organized in Japan every year since 1974, and a symposium on food hydrocolloids (http://food.hydrocolloids.org/) in Japan has been organized every year since 1990. Food hydrocolloids have been widely used in the food industry for their gelling, thickening, emulsifying, dispersing, and stabilizing functions. They have recently attracted much attention for controlling the food texture for elderly people having the difficulty in mastication and deglutition, and also as their functions as dietary fiber, which is expected to lower the cholesterol and blood pressure thus preventing lifestyle related disease. Food hydrocolloids control the texture and at the same time, they control the flavor and aroma release, which has also been studied extensively. Methods of investigation are very diverse, ranging from physics to biological chemistry, and workers in this field must master various methods. Japanese Society of Food Hydrocolloids organizes an introductory seminar held the day before the hydrocolloids symposium. The Society of Rheology, Japan (http://wwwsoc.nii. ac.jp/srj/) has organized a short course of food rheology for beginners every year since 2002 .

Japan Association of konjac (http://www.konnyaku.or.jp) was established in 1963 by representatives of farmers, and manufacturers of konjac powders and various konjac products. This association organizes cooking schools and promotion of konjac consumption, as well as activating research on the beneficial effect of konjac as a source of dietary fiber.

Acknowledgement This research was partially supported by the Ministry of Education, Science, Sports and Culture, Grant-in-Aid for Scientific Research (A) 19200051. The author wishes to thank São Paulo University for the invitation to Japan-Brazil Memorial Symposium on Science and Technology 1908-2008, 100 Years of Japanese Immigration to Brazil.

\section{References}

Annable, P., Williams, P.A. and Nishinari, K. (1994). Interaction in xanthan- glucomannan mixtures and the influence of electrolyte. Macromolecules, 27, 4204-4211.

Arai, S. (2007). Functions of food and health- The present situation and the future. Nihon Eiyou Shokuryou Gakkaishi, 60(6), M64-69 (In Japanese).

Bourne, M. (2002). "Food Texture and Viscosity, Concept and Measurement", $2^{\text {nd }}$ Ed., Academic Press, N.Y.

Funami, T., Kataoka, Y., Omoto, T., Goto, Y., Asai, I. and Nishinari, K. (2005). Food Hydrocolloids control the gelatinization and retrogradation behavior of starch. $2 \mathrm{~b}$. Functions of guar gums with different molecular weights on the retrogradation behavior of corn starch. Food Hydrocolloids, 19, 25-36.

Gao, S. and Nishinari, K. (2004). Effect of degree of acetylation on gelation of konjac glucomannan. Biomacromolecules, 5, 175-185.

Gidley, M and Nishinari, K.(2009). Physico-chemistry, in "Chemistry, Biochemistry and Biology of 1,3 $\beta$ Glucans", ed by A. Bacic, G.B. Fincher and B A Stone, Elsevier, in press.

Harada, T., Masada, M., Fujimori, K. and and Maeda, I. (1966). 
Production of a firm, resilient gel-forming polysaccharide by a mutant of Alcaligenes faecalis var. myxogenes $10 \mathrm{C} 3$. Agr. Biol. Chem., 30, 196-198.

Hayakawa, F., Ioku, K., Akuzawa, S., Saito, M., Nishinari, K., Yamano, Y. and Kohyama, K. (2005). Collection of Japanese texture terms (Studies on Japanese texture terms Part1). Nippon Shokuhin Kagaku Kogaku Kaishi, 52, 337-346 (In Japanese with English summary).

Huang, L., Takahashi, R., Kobayashi, S., Kawase, T. and Nishinari, K. (2002). Gelation behavior of native and acetylated konjac glucomannan. Biomacromolecules, 3, 1296-1303.

Hutchings, J.B. and Lillford, P.L. (1988). The perception of food texture- the philosophy of the breakdown path. J. Texture Studies, 19, 103-115

Kawai, S., Nitta, Y. and Nishinari, K. (2008). Model study for large deformation of physical polymeric gels. J. Chem. Phys., 128, 134903.

Kiho, T., Kochi, M., Usui, S., Hirano, K., Aizawa, K., Inakuma, T. (2001). Effect of an acidic polysaccharide. (TAP) from Tremella aurantia and its degradation product (TAP-H). Biol. Pharm. Bull., 24, 1400-1403.

Kim, B. S., Takemasa, M. and Nishinari, K. (2006). Synergistic interaction of xyloglucan and xanthan investigated by rheology, differential scanning calorimetry, and NMR. Biomacromolecules, 7, 1223-1230.

Kohyama, K., Iida, H. and Nishinari, K. (1993). A mixed system composed of different molecular weights konjac glucomannan and kappa-carrageenan: large deformation and dynamic viscoelastic study. Food Hydrocolloids, 7, 213-226.

Lee, C-K., Klopp, R.G., Weindruch, R. and Prolla, T.A. (1999). Gene expression profile of aging and its retardation by caloric restriction. Science, 285, 1390-1393.

Logemann, J. (1983). "Manual for the Videofluoroscopic Study of Swallowing" ( $2^{\text {nd }}$ Ed.,) Pro-Ed. Inc.

Maekaji, K. (1978). Determination of acidic component of konjac mannan. Agric. Biol. Chem., 42, 177-178.

Maekaji, K. and Kawamura, D. (1984). Relationship between stress relaxation and syneresis of konjac mannan gel. Agric. Biol. Chem., 48, 227-228.

Matsuo, M., Takaya, T., Miwa, S., Moritalka, H. and Nishinari, K. (2002). The uniaxial compression test as a simulation of mastication for the texture evaluation of cooked rice. J. Jap. Soc. Mastication Science Health Promotion, 12, 11-25

Michiwaki, Y., Yokoyama, M., Kinumatsu, Y., Mori, K. and Michi, K. (2002). Cine MRI to demonstrate normal swallow. J. Jpn. Stomatol. Soc., 51, 237-2.43 (In Japanese with English summary).

Miyoshi, E., Takaya, T. and Nishinari, K. (1998). Effects of glucose, mannose and konjac glucomannan on the gel-sol transitiom in gellan gum aqueous solutions by rheology and DSC. Polymer Gels \& Networks, 6, 273-290.

Momose,T., Nishikawa, J., Watanabe,T., Sasaki,Y., Senda, M., Kubota, K., Sato, Y., Funakoshi, M., and Minakuchi, S. (1997). Effect of mastication on regional cerebral blood flow in humans examined by positron-emission tomography with ${ }^{15} \mathrm{O}$-labelled water and magnetic resonance imaging. Archives Oral Biol., 42, 57-61.

Niki, R., Kohyama, K., Sano, Y. and Nishinari, K. (1994). Rheological study on the rennet-induced gelation of casein micelles with different sizes. Polymer Gels \& Netwks, 2, 105-118.

Nishinari, K., Horiuchi, H., Ishida, K., Ikeda, K., Date, M. and Fukada, E. (1980). A new apparatus for rapid and easy measurement of dynamic viscoelasticity for gel-like foods. Nippon Shokuhin Kogyo Gakkaishi, 27, 227-233 (In Japanese with English summary).

Nishinari, K., Koide, S. and Ogino, K. (1985). On the temperature dependence of elasticity of thermoreversible gels. J. Physique (France), 46, 793-797.

Nishinari, K. (1987). Food Hydrocolloids in Japan, in "Gums and Stabilisers for the Food Industry 4", ed by G.O.Phillips, D.J.Wedlook \& P.A.Williams, IRL Press, Oxford, pp. 373-390.

Nishinari, K. (2000). Konjac glucomannan, in "Novel Macromolecules in Food Systems", ed. by G. Doxastakis and V. Kiosseoglou, Elsevier Science B.V., Amsterdam, pp. 309-330.
Nishinari, K. (2004). Rheology, food texture and mastication. in Special issue on Mastication and Food Texture, Ed. Nishinari, K. J. Texture Stud., 35, 113-124.

Nishinari, K. Ed. (2007). "Development and Application of Food Hydrocolloids", (In Japanese), CMC Publications, Tokyo.

Nishinari, K. (2005). Hydrocolloids, in "Biotechnological Applications of Marine Natural Substances", ed. by N. Fusetani (In Japanese), CMC Publications, Tokyo, pp. 238-259.

Nishinari, K., Kohyama, K., Ying, Z., Kitamura, K., Sugimoto, T., Saio, K. and Kawamura, Y. (1991). Rheological study on the effect of the A5 subunit on the gelation characteristics of soybean protein. Agric. Biol. Chem., 55, 351-355.

Nishinari, K., Okoshi, H., Kohyama, K., Yamamoto,T., Eds. (2005) "Handbook of Creation of New Texture" (In Japanese), Science Forum Publ., Tokyo.

Nishinari, K. Hayakawa, F., Xia, C-F., Huang, L., Meullenet, J-F. and Sieffermann, J-M. (2008). Comparative study of texture terms: English, French, Japanese, and Chinese. J. Texture Stud. 39, 530-568.

Nitta, Y., Kim, B. S., Nishinari, K, Shirakawa, M., Yamatoya, K., Oomoto, T. and Asai, I. (2003). Synergistic gel formation of xyloglucan/gellan mixture as studied by rheology, DSC and circular dichroism. Biomacromolecules, 4, 1654-1660.

Nitta, Y., Fang, Y., Takemasa, M., and Nishinari, K. (2004). Gelation of xyloglucan by addition of epigallocatechin gallate as studied by rheology and calorimetry. Biomacromolecules, $\mathbf{5}$ 1206-1213.

Okadome, H., Toyoshima, H., Sudo, M., Ando, I., Numaguchi, K., Horisue, N. and Ohtsubo, K. (1998). Palatability evaluation for Japonica rice grains based on multiple physical measurements of individual cooked rice grain. Nippon Shokuhin Kogyo Gakkaishi, 45, 398-407 (In Japanese with English summary).

Onozuka, M., Fujita, M., Watanabe, K., Hirano, Y., Niwa, M., Nishiyama, K., Saito, S. (2003). Age-related changes in brain regional activity during chewing: A functional magnetic resonance imaging study. J. Dental Res., 82, 657-660.

Pongsawatmanit, R., Temsiripong, T., Ikeda, S. and Nishinari, K. (2006). Influence of tamarind seed xyloglucan on rheologica properties and thermal stability of tapioca starch. J. Food Engineering, 77, 41-50.

Shiba, K. and Nishinari, K. (2007). Seaweed polysaccharides, in "Development and Application of Food Hydrocolloids", ed by K.Nishinari (In Japanese), CMC Publications, Tokyo, pp. 196-211.

Szczesniak, A.S. (2002). Texture is a sensory property. Food Quality and Preference, 13, 215-225.

Uwabe, M. and Nishinari, K. (2001). Effects of the addition of mocal paste to bread on the textural and nutritional properties. $J$. Integrated Study Dietary Habits, 12, 53-61 (In Japanese).

Yamori, Y. (2007). Dietary pattern in the world and the longevityLongevity in the world and in Japan. Nihon Eiyou Shokuryou Gakkaishi, 60(6), M58-64 (In Japanese).

Yoshikawa, S., Nishimaru, S., Tashiro, T. and Yoshida, M. (1970). Collection and classification of words for description of food texture. :Collection of words. J. Texture Stud., 1, 437-442.

Yoshimura,M., Naito, S., Nagano, T. and Nishinari, K. (2007). Effect of concentration of soybean powder on the rheological properties and the network structure of soybean curd prepared from powdered soybean. Nippon Shokuhin Kagaku Kogaku Kaishi, 54, 143-151 (In Japanese with English summary and figure captions).

Yoshimura,M., Shibata, F., Eto, M. and Nishinari, K. (2004). Rheological studies on gelation process of dispersion of powdered soybean in the presence of glucono- $\delta$ - lactone. Nippon Shokuhin Kagaku Kogaku Kaishi, 51, 115-122 (In Japanese with English summary and figure captions)

Yoshimura, M., Takaya, T. and Nishinari, K. (1996). Effects of konjac-glucomannan on the gelatinization and retrogradation of corn starch by rheology and differential scanning calorimetry. J. Agric. Food Chem., 44, 2970-2976.

Yoshimura, M., Takaya, T. and Nishinari, K. (1999). Effects of xyloglucan on the gelatinization and retrogradation of corn starch as studied by rheology and differential scanning calorimetry. Food Hydrocolloids, 13, 101-111. 